\title{
Diversidade e similaridade entre populações de insetos aquáticos em riachos de primeira e segunda ordem, sul do Brasil
}

\author{
Similarity and diversity between aquatic insect populations in \\ streams of first and second order, south of Brazil
}

\author{
Cibele Bender Raioํ, Andréia Avian Espinoza², Sirlei Terezinha Bennemann ${ }^{3}$
}

\section{Resumo}

A estrutura das comunidades de macroinvertebrados aquáticos é influenciada pela velocidade da correnteza do curso d'água e da heterogeneidade de habitats entre outras características. Este trabalho verificou a relação da diversidade de insetos aquáticos com a ordem hidrológica de dois riachos. Para isso, foi testada a seguinte hipótese: a diversidade de insetos associados ao mesmo substrato em trechos equivalentes de riachos classificados em diferentes ordens é semelhante. Amostras de folhiço foram coletas nos trechos médios do riacho Cascatinha (primeira ordem) e do riacho João Pinheiro (segunda ordem), pertencentes à bacia do rio Tibagi, Telêmaco Borba, Paraná, Brasil. A diversidade de cada riacho foi obtida pelo índice de Shannon. Os valores do índice de diversidade foram baixos para ambos os riachos $(0,8$ no riacho de primeira ordem e 0,89 para o riacho de segunda ordem), não havendo diferença estatística $(t=-1,12 ; p=0,27)$. A similaridade entre os riachos foi de 0,51 . Esses resultados permitiram verificar que, nos riachos estudados, não há efeito da ordem hidrológica sobre a diversidade de insetos aquáticos $(\mathrm{F}=0,14 ; \mathrm{p}=0,71)$, assim, a hipótese testada foi aceita. $\mathrm{O}$ táxon mais abundante, em ambos os riachos, foi Chironomidae (Diptera), representando $82,7 \%$ do total de indivíduos coletados. No entanto, ao retirar-se Chironomidae das análises, a hipótese passa a ser rejeitada. A elevada abundância de indivíduos de Chironomidae influenciou os valores da diversidade local.

Palavras-chave: Chironomidae. Folhiço. Invertebrados. Microbacias.

\begin{abstract}
Aquatic communities of macroinvertebrates are influencied by rapid velocity, habitat heterogeneity and other characteristics. This study examined a relationship between aquatic insects diversity with hydrologic order of two streams. We tested following hypothesis: diversity of insects associated with the same substrate in equal parts of streams, classified in different orders, are similar. Samples of litter were collected in middle stretches of stream Cascatinha (first order) and stream João Pinheiro (second order), Tibagi river basin, Telêmaco Borba, Paraná, Brazil. Diversity of each stream was obtained by Shannon index. Diversity values were low for both streams $(0.8$ for the first order stream and 0.89 for the second order stream) with no statistical difference $(t=-1.12 ; p=0.27)$. Similarity between the streams was 0.51 . These results allowed us to verify that, to studied streams, hydrological order has no effect
\end{abstract}

1 Aluna do Programa de Pós-Graduação em Ciências Biológicas (Zoologia) da Universidade Estadual de Londrina. Laboratório de Ecologia Trófica, Departamento de Ciências Animal e Vegetal, Centro de Ciências Biológicas. cibele_bio@hotmail.com

2 Aluna do Curso de Ciências Biológicas da Universidade Estadual de Londrina. Laboratório de Ecologia Trófica, Departamento de Ciências Animal e Vegetal, Centro de Ciências Biológicas. ae@hotmail.com

3 Docente do Programa de Pós-Graduação em Ciências Biológicas (Zoologia) da Universidade Estadual de Londrina. Laboratório de Ecologia Trófica, Departamento de Ciências Animal e Vegetal, Centro de Ciências Biológicas. sirlei@uel.br 
on aquatic insects diversity $(\mathrm{F}=0.14, \mathrm{p}=0.71)$, so the hypothesis was accepted. The most abundant taxon in both streams was Chironomidae (Diptera), representing $82.7 \%$ of total collected. However when Chironomidae was removed from analysis, the hypotesis was refused. An high abundance of Chironomidae individuals had influenced values of local diversity.

Keywords: Chironomidae. Invertebrates. Litter. Watershad.

\section{Introdução}

Riachos são definidos por seu curso d'água unidirecional, com pequena profundidade, volume de água e ordem mais baixa que os rios. Segundo a Classificação Hidrológica de Straher (1957), um riacho que não recebe afluente é classificado como de primeira ordem, o riacho que recebe o de primeira ordem é um riacho de segunda ordem e assim por adiante. Desse modo, um riacho pode ter trechos de diferentes ordens, de acordo com os afluentes e subafluentes que recebem.

Estudos de ecologia em ambientes aquáticos demonstram que a distribuição dos invertebrados nestes ambientes está relacionada ao tipo de substrato (KIKUCHI; UIEDA, 2005), à integridade da mata ciliar (GALVES; JEREP; SHIBATTA, 2007), à pluviosidade (GOLÇALVES; ARANHA, 2004), além de outros fatores físicos, como a velocidade da correnteza (KIKUCHI; UIEDA, 1998; REZENDE, 2007).

A crescente modificação dos ambientes naturais e a atual degradação dos ecossistemas tornam urgentes os estudos em ambientes aquáticos. As atividades humanas levam à uma necessidade de manejo e reestruturação dos ambientes. O levantamento e o conhecimento das espécies que compõe a fauna de invertebrados, bem como a definição da estrutura dessas comunidades em riachos íntegros, ou pouco alterados, são conhecimentos base para a aplicação de ações de restauração e manejo desses ambientes.

O presente trabalho teve por objetivo verificar a relação da diversidade da entomofauna aquática encontrada no substrato folhiço em riachos de diferentes ordens, ambos localizados em área de conservação. Para tanto, a seguinte hipótese foi testada: insetos aquáticos associados ao mesmo substrato, em trechos da porção média de riachos de diferentes ordens, têm diversidade semelhante.

\section{Material e Métodos}

\section{Área de estudo}

Foram amostrados dois riachos que nascem no Parque Ecológico da Fazenda Monte Alegre (Figura 1), uma Reserva Ecológica pertencente à empresa Klabin S.A. de Papel e Celulose. A fazenda está localizada no município de Telêmaco Borba, no estado do Paraná $\left(24^{\circ} 12^{\prime} 42^{\prime \prime}\right.$ e $\left.50^{\circ} 33^{\prime} 26^{\prime} \mathrm{W}\right)$, e possui uma área de 126 mil hectares, dos quais 46 mil são de áreas de preservação que se caracterizam como floresta ombrófila mista, floresta estacional semi-decídua e campos naturais, com altitude média de $885 \mathrm{~m}$. O clima, segundo a classificação de Köeppen-Geiger, é subtropical, transicional para o temperado, úmido, mesotérmico, sem estação seca definida (Cfa/Cfb). (AZEVEDO et al., 2008). 

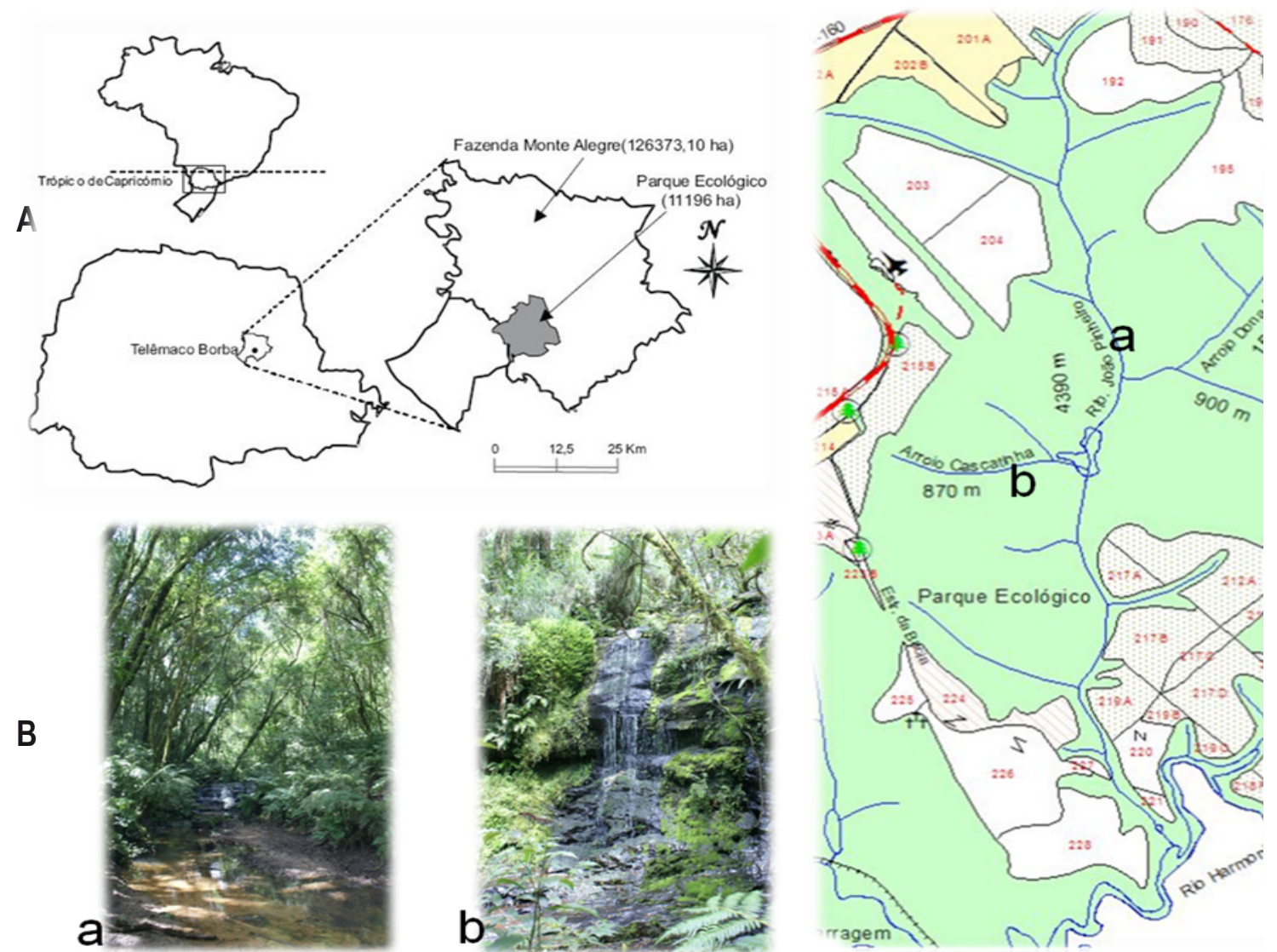

Figura 1. (A) Localização da área de estudo (Fonte: adaptado de Reis et al. (2006)). (B) Trecho médio dos riachos amostrados. (a) riacho João Pinheiro e (b) riacho Cascatinha. (C) Microbacia do riacho João Pinheiro (Fonte: Acervo Klabin).

O riacho de primeira ordem, denominado Cascatinha, deságua no riacho João Pinheiro, em seu trecho médio, que é de segunda ordem. Ambos estão em uma área de mata secundária e possuem fundo semelhante composto por cascalho, areia e argila. O riacho Cascatinha possui $870 \mathrm{~m}$ de extensão, enquanto o ribeirão João Pinheiro, $4.390 \mathrm{~m}$.

As variáveis físicas e químicas (temperatura, condutividade elétrica, oxigênio dissolvido e $\mathrm{pH}$ ) foram obtidas através de aparelhos eletrônicos portáteis, sempre antes de serem iniciadas as amostragem. A velocidade da água foi calculada pelo tempo que um flutuador percorria uma distância pré-determinada, a profundidade e a largura do riacho foram medidas com trena (precisão $1 \mathrm{~mm})$. Para o cálculo da vazão multiplicou-se a velocidade, profundidade e largura média (as médias foram obtidas a partir de 10 mensurações de cada parâmetro).

O método de amostragem foi adaptado de Bennemann e Galves (2008): o substrato foi rastelado em direção a uma peneira $(90 \mathrm{~cm}$ e malha $2 \mathrm{~mm}$ ) disposta perpendicularmente ao curso d'água, imediatamente a jusante a peneira era disposta uma rede D $(500 \mu \mathrm{m})$, assim o folhiço e os insetos de 
maior porte ficavam retido na malha, enquanto partículas e insetos menores, que atravessavam a malha da peneira, eram coletados em rede $\mathrm{D}$ $(500 \mu \mathrm{m})$. Em cada riacho, foram coletadas duas amostras de folhiço (com aproximadamente 90 cm de diâmetro cada), uma amostra obtida em área de poção e a outra em área de corredeira. A distância entre cada amostragem, em cada um dos riachos, era de $100 \mathrm{~m}$. As coletas foram realizadas em março de 2008 durante a disciplina Biologia de Campo do Curso de Mestrado em Ciências Biológicas da Universidade Estadual de Londrina (UEL).

O folhiço e as amostras da rede $\mathrm{D}$, depois de fixadas em etanol $70 \%$, foram levados ao laboratório de ecologia trófica UEL. Após a triagem, os animais foram identificados em nível taxonômico "família", com o auxílio de um microscópio estereoscópico Heerbrugg Switzerland Wind M3Z e dos manuais de identificação de Pérez (1988), Costa, Ide e Simonka (2006) e Nalim et al. (2008). O material coletado foi depositado no laboratório de ecologia trófica da UEL.

\section{Análise dos Dados}

Para a comparação e tratamento estatístico dos resultados, os dados das duas amostragens em cada riacho foram agrupados. As análises estatísticas foram obtidas com o auxílio do software PAST (Palaeontological Statistics ver. 1.8). A diversidade biológica foi calculada com a utilização do Índice de Diversidade de Shannon (H') e a similaridade foi analisada seguindo os Índices de Sörensen. A comparação dos dois riachos foi realizada pela análise da variância.

\section{Resultados e Discussão}

As variáveis abióticas analisadas durante as amostragens são apresentadas na Tabela 1 . O riacho João Pinheiro apresentou maior vazão e velocidade da correnteza, provavelmente relacionadas à maior ordem desse riacho.

Tabela 1. Dados abióticos dos riachos estudados, média e desvio padrão (DP).

\begin{tabular}{lcccc}
\hline Variável & Riacho João Pinheiro & Riacho Cascatinha & Média & DP \\
\hline Temperatura $\left({ }^{\circ} \mathrm{C}\right)$ & 21 & 21 & 21 & 0 \\
Velocidade $\left({\left.\mathrm{m} \cdot \mathrm{s}^{-1}\right)}^{2,4}\right.$ & 2,2 & 2,3 & 0,14 \\
Vazão $\left(\mathrm{m}^{3} \cdot \mathrm{s}^{-1}\right)$ & 1,42 & 0,08 & 0,75 & 0,95 \\
Cond. Elétrica $\left(\mathrm{mS} \cdot \mathrm{cm}^{-1}\right)$ & 21 & 32 & 26,5 & 7,78 \\
$\mathrm{O}^{2}$ dissolvido & 6,5 & 7,44 & 6,97 & 0,66 \\
$\mathrm{pH}$ & 5,32 & 5,32 & 5,32 & 0 \\
\hline
\end{tabular}

Foram capturados 1.484 indivíduos, sendo 557 no riacho Cascatinha e 927 no riacho João Pinheiro, pertencentes a oito ordens e vinte famílias de insetos (Apêndice I).

O índice de diversidade de Shannon foi de 0,8 no riacho Cascatinha e 0,89 para o riacho João
Pinheiro, não diferindo significativamente ( $\mathrm{t}=$ $1,12 ; p=0,27)$. Pela análise do índice de Sörensen, verificou-se entre os riachos, uma similaridade de 0,51. A análise da variância $(F=0,14 ; p=0,71)$ permitiu verificar que não há efeito da ordem do riacho sobre a diversidade de insetos aquáticos. 
Os representantes das famílias de insetos encontrados neste estudo também foram encontrados por Nalim et al. (2008) em um levantamento realizado no Ribeirão Varanal, um riacho íntegro, com trechos de primeira e segunda ordem, afluente do rio Tibagi, localizado próximo à micro-bacia do João Pinheiro. Os autores supracitados encontraram maior riqueza e alguns organismos que não foram registrados no presente estudo, como Diptera-Dixidae, Ephemeroptera-Leptohyphidae, PlecopteraGripopterygidae, entre outros.

Entre os representantes das 22 famílias encontradas no substrato folhiço do Ribeirão Varanal, 17 correspondem aos mesmos táxons registrados neste estudo. A maior riqueza encontrada naquele ribeirão se deve, em parte, ao fato de terem sido colhidas amostras nos diferentes trechos de sua extensão: nascente, médio e foz.

A maior abundância, no riacho Cascatinha e no riacho João Pinheiro foi de DipteraChironomidae, representando $82,7 \%$ do total de amostras (Figura 2). O Índice H' está relacionado com a abundância de indivíduos, logo a elevada abundância dessas larvas foi responsável pelo baixo valor da diversidade. A elevada ocorrência de indivíduos desta família também foi encontrada em outros estudos em riachos tropicais (e.g. KIKUCHI; UIEDA, 1998; RIBEIRO; UIEDA, 2005; DAVANSO; HENRY, 2007). As formas imaturas de Chironomidae estão especialmente associadas ao substrato folhiço, pois utilizam as partículas finas para a construção de túbulos e para alimentação (KIKUCHI; UIEDA, 2005).

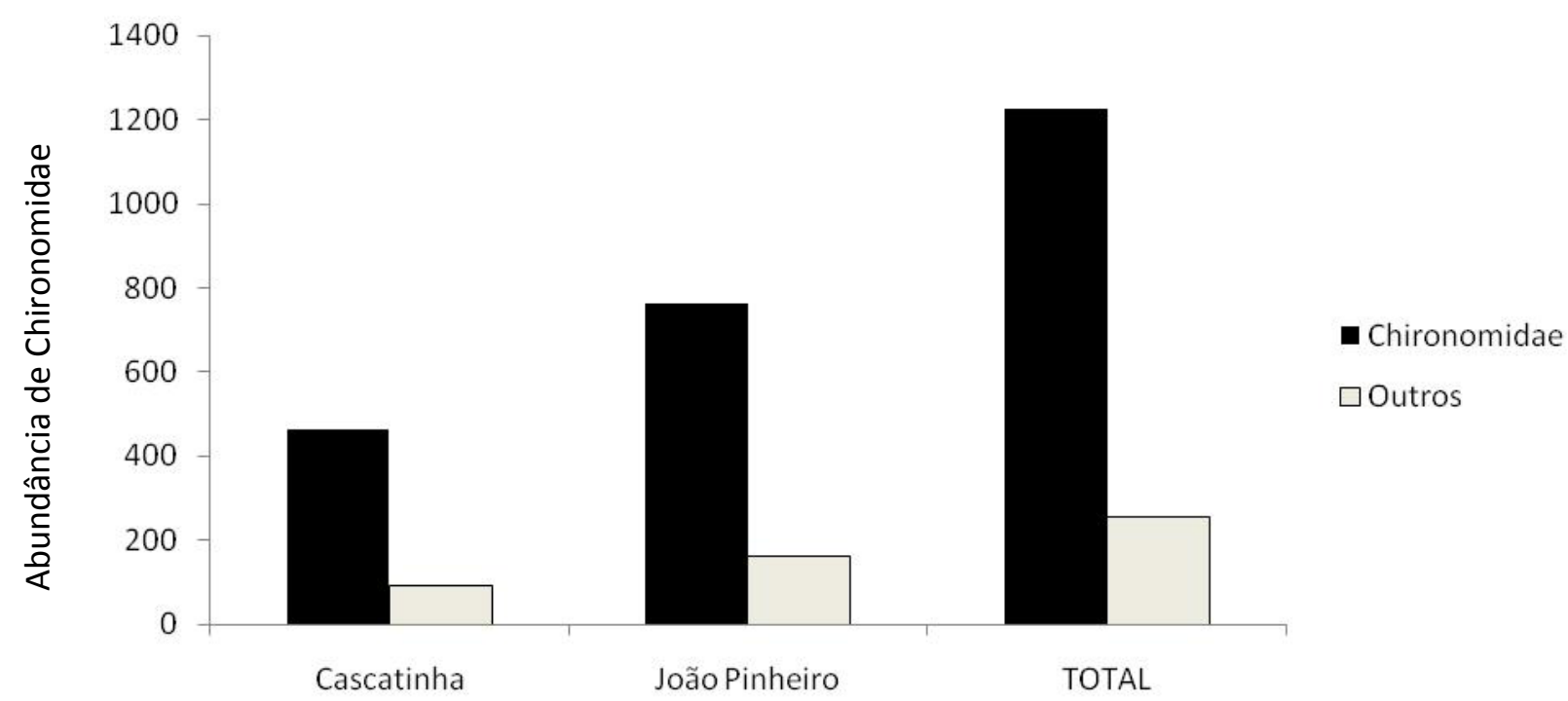

Figura 2. Abundância de indivíduos representantes da família Chironomidae, ordem Diptera, em relação às outras famílias de insetos amostrados nos riachos João Pinheiro e Cascatinha.

Os resultados aqui apresentados permitem verificar que houve variação da diversidade da comunidade de insetos entre os riachos de ordens diferentes, porém a diferença apresentada pela diversidade de Shannon não foi estatisticamente significativa. Assim, a hipótese de que a diversidade de insetos associados ao mesmo substrato em trechos equivalentes de riachos classificados em 
diferentes ordens é semelhante, foi aceita para esses riachos.

Araújo-Lima et al. (1999), ao estudar a riqueza de peixes em riachos de diferentes ordens, também não verificou uma relação entre a riqueza e a ordem do riacho e os autores afirmam que uma maior riqueza está associada à diversificação de substratos e não à ordem. Para insetos aquáticos, a estruturação das comunidades também parece ser mais influenciada pelas condições ambientais do que pela localização espacial (COSTA; MELO, 2008).
No entanto, quando retirado das análises os representantes da família Chironomidae (Figura 3), a diversidade aumenta para 2,03 para o riacho Cascatinha e 2,39 para o riacho João Pinheiro, ou seja, significativamente maior para o riacho de maior ordem $(t=-2,9 ; p=0,0037)$. A grande abundância de representantes da família Chironomidae tem elevado peso para as análises. Desconsiderando a família Chironomidae a hipótese é rejeitada, indicando que há influência da ordem na diversidade de insetos nos riachos estudados.

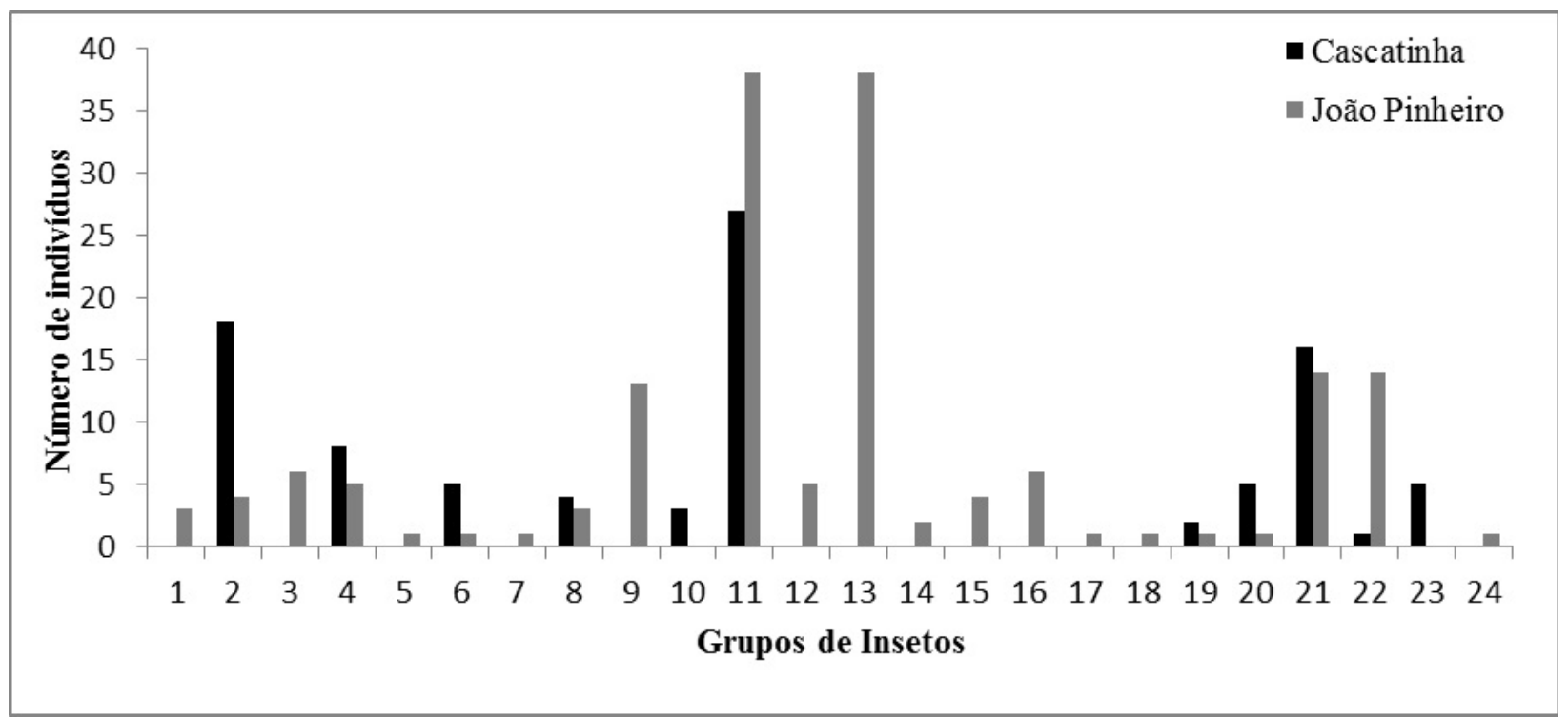

Figura 3. Abundância dos representantes das famílias de insetos coletados no riacho João Pinheiro e no riacho Cascatinha. 1.Coleoptera Elmidae; 2. Coleoptera Ptilodactylidae; 3. Coleoptera - Não identificado; 4. Diptera Ceratopogonidae; 5. Diptera Culicidae; 6. Diptera Empididae; 7. Diptera Muscidae; 8. Diptera Simuliidae; 9 . Diptera Não identificado; 10. Ephemeroptera Baetidae; 11. Ephemeroptera Leptophlebiidae; 12. Ephemeroptera Tricorythidae; 13. Ephemeroptera - Não identificada; 14. Hemiptera Belostomatidae; 15. Odonata - Calopterygidae; 16. Odonata Coenagrionidae; 17. Odonata Gomphidae; 18. Odonata Libellulidae; 19. Odonata - Não identificada; 20. Plecoptera Perlidae; 21. Trichoptera Hydropsychidae; 22. Trichoptera Leptoceridae; 23. Trichoptera - Não identificada; 24. Megaloptera Corydalidae.

\section{Agradecimentos}

Agradecemos ao Programa de Mestrado em Ciências Biológicas da Universidade Estadual de Londrina; à empresa Klabin S.A. de Papel e Celulose; à CAPES pela bolsa concedida à primeira autora; à Msc. Andréa Martine Ribeiro e Raphael
Lou Tchog Ruiz pelo auxílio nas coletas e à Msc. Taciana Lopes Coppo pelas discussões dos dados.

\section{Referências}

ARAÚJO-LIMA， C. A. R. M.; JIMÉNEZ, L. F.; OLIVEIRA, R. S.; ETEROVICK, P. C.; MENDONZA, 
U.; JEROZOLIMKI, A. Relação entre o número de espécies de peixes, complexidade de hábitat e ordem do riacho nas cabeceiras de um tributário do rio Urubu, Amazônia Central. Acta Limnologica Brasiliensia, Botucatu, v. 11, n. 2, p. 127-135, 1999.

AZEVEDO, T. I. N.; SEKIAMA, M. L.; VIEIRA, A. O. S.; BENNEMANN, S. T. Descrição física da micro bacia do ribeirão Varanal e caracterização dos trechos. In: BENNEMANN, S. T.; SHIBATTA, O. A.; VIEIRA, A. O. S. A flora e a fauna do Ribeirão Varanal: um estudo da biodiversidade no Paraná. Londrina: EDUEL, 2008. p. 7-14.

BENNEMANN, S. T.; GALVES, W. Metodologia de amostragem da fauna aquática. In: BENNEMANN, S. T.; SHIBATTA, O. A.; VIEIRA, A. O. S. A flora e a fauna do Ribeirão Varanal: um estudo da biodiversidade no Paraná. Londrina: EDUEL, 2008. p. 69-75.

COSTA, C.; IDE, S.; SIMONKA, C. E. (Ed.). Insetos imaturos: metamorfose e identificação. Ribeirão Preto: Holos, 2006.

COSTA, S. S.; MELO, A. S. Beta diversity in stream macroinvertebrate assemblages: among-site and amongmicrohabitat components. Hydrobiologia, The Hague, v. 598, p. 131-138, 2008.

DAVANSO, R. C. S.; HENRY, R. Composition and abundance of Chironomidae and Ephemeroptera in a lateral lake in the mouth zone of Paranapanema River into Jurumirim Reservoir (State of São Paulo). Acta Limnologica Brasiliensia, Botucatu, v. 19, n. 2, p. 131142, 2007.

GALVES, W.; JEREP, F. C.; SHIBATTA, O. A. S. Estudo da condição ambiental pelo levantamento da fauna de três riachos na região do Parque Estadual Mata dos Godoy (PEMG), Londrina, PR, Brasil. Pan-American Journal of Aquatic Sciences, v. 2, n. 1, p. 55-65, 2007.

GONÇALVES, F. B.; ARANHA, J. M. R. Ocupação espaço-temporal pelos macroinvertebrados bentônicos na bacia do rio Ribeirão, Paranaguá, PR (Brasil). Acta Biológica Paranaense, Curitiba, v. 33, n. 1-4, p. 181-191, 2004.

KIKUCHI, R. M.; UIEDA, V. S. Composição da comunidade de invertebrados de um ambiente lótico tropical e sua variação espacial e temporal. In: NESSIMIAN, J. L.; CARVALHO, A. L. Ecologia de insetos aquáticos. Rio de Janeiro: Série Oecologia Brasiliensis, 1998. p. 157-173.

KIKUCHI, R. M.; UIEDA, V. S. Composição e distribuição dos macroinvertebrados em diferentes substratos de fundo de um riacho no município de Itatinga, São Paulo, Brasil. Entomología y Vectores,
Salta, v. 12, n. 2, p. 193-231, 2005.

NALIM, D. M.; GALVES JUNIOR, W.; MENDES, E. C.; MARONEZE, D. M. Insetos aquáticos. In: BENNEMANN, S. T.; SHIBATTA, O. A.; VIEIRA, A. O. S. A flora e a fauna do Ribeirão Varanal: um estudo da biodiversidade no Paraná. Londrina: EDUEL, 2008. p. 111-138.

PÉREZ, G. R. Guía para el estudio de los macroinvertebrados acuáticos del departamento de Antioquia: universidad de Antioquia. Bogotá: Presencia, 1988.

REIS, N. R.; PERACCHI, A. L.; LIMA, I. P.; PEDRO, W. A. Riqueza de espécies de morcegos (Mammalia, Chiroptera) em dois diferentes habitats, na região centro-sul do Paraná, sul do Brasil. Revista Brasileira de Zoologia, Curitiba, v. 23, n. 3, p. 813-816, set. 2006.

REZENDE, C. F. Estrutura de comunidade de macroinvertebrados associados ao folhiço submerso de remanso e correnteza em igarapés da Amazônia Central. Biota Neotropica, Campinas, v. 7, n. 2, p. 301-305, 2007.

RIBEIRO, L. O.; UIEDA, V. S. Estrutura da comunidade de macroinvertebrados bentônicos de um riacho de serra em Itatinga, São Paulo, Brasil. Revista Brasileira de Zoologia, Curitiba, v. 22, n. 3, p. 613-618, 2005.

STRAHLER, H. N. Quantitative analysis of wathersheg geomorfhology. Transactions American Geophysical Union, Washington, v. 38, p. 913-920, 1957.

Recebido em 10 de dezembro de 2009 - Received on December 10, 2009

Aceito em 16 de março de 2010 - Accepted on March 16, 2010 
Apêndice 1. Abundância em número de indivíduos das famílias de insetos encontrados folhiço dos riachos João Pinheiro e Cascatinha.

\begin{tabular}{|c|c|c|c|c|c|}
\hline Taxa & Cascatinha & João Pinheiro & TOTAL & Média & DP \\
\hline \multicolumn{6}{|l|}{ Ordem Coleoptera } \\
\hline Elmidae & 0 & 3 & 3 & 1,5 & 2,12 \\
\hline Ptilodactylidae & 18 & 4 & 22 & 11 & 9,9 \\
\hline Não identificada & 0 & 6 & 6 & 3 & 4,24 \\
\hline \multicolumn{6}{|l|}{ Ordem Diptera } \\
\hline Ceratopogonidae & 8 & 5 & 13 & 6,5 & 2,12 \\
\hline Chironomidae & 463 & 764 & 1227 & 613,5 & 212,83 \\
\hline Culicidae & 0 & 1 & 1 & 0,5 & 0,71 \\
\hline Empididae & 5 & 1 & 6 & 3 & 2,83 \\
\hline Muscidae & 0 & 1 & 1 & 0,5 & 0,71 \\
\hline Simuliidae & 4 & 3 & 7 & 3,5 & 0,71 \\
\hline Não identificada & 0 & 13 & 13 & 6,5 & 9,19 \\
\hline \multicolumn{6}{|c|}{ Ordem Ephemeroptera } \\
\hline Baetidae & 3 & 0 & 3 & 1,5 & 2,12 \\
\hline Leptophlebiidae & 27 & 38 & 65 & 32,5 & 7,79 \\
\hline Tricorythidae & 0 & 5 & 5 & 2,5 & 3,54 \\
\hline Não identificada & 0 & 38 & 38 & 19 & 26,87 \\
\hline \multicolumn{6}{|l|}{ Ordem Hemiptera } \\
\hline Belostomatidae & 0 & 2 & 2 & 1 & 1,41 \\
\hline \multicolumn{6}{|l|}{ Ordem Odonata } \\
\hline Calopterygidae & 0 & 4 & 4 & 2 & 2,83 \\
\hline Coenagrionidae & 0 & 6 & 6 & 3 & 4,24 \\
\hline Gomphidae & 0 & 1 & 1 & 0,5 & 0,71 \\
\hline Libellulidae & 0 & 1 & 1 & 0,5 & 0,71 \\
\hline Não identificada & 2 & 1 & 3 & 1,5 & 0,71 \\
\hline \multicolumn{6}{|l|}{ Ordem Plecoptera } \\
\hline Perlidae & 5 & 1 & 6 & 3 & 2,83 \\
\hline \multicolumn{6}{|l|}{ Ordem Trichoptera } \\
\hline Hydropsychidae & 16 & 14 & 30 & 15 & 1,4 \\
\hline Leptoceridae & 1 & 14 & 15 & 7,5 & 9,19 \\
\hline Não identificada & 5 & 0 & 5 & 2,5 & 3,53 \\
\hline \multicolumn{6}{|l|}{ Ordem Megaloptera } \\
\hline Corydalidae & 0 & 1 & 1 & 0,5 & 0,71 \\
\hline TOTAL & 557 & 927 & 1484 & & \\
\hline
\end{tabular}

